PROCEEDINGS OF THE

AMERICAN MATHEMATICAL SOCIETY

Volume 134, Number 5, Pages 1495-1498

S 0002-9939(05)08097-4

Article electronically published on October 4, 2005

\title{
COMPUTABLE TREES, PRIME MODELS, AND RELATIVE DECIDABILITY
}

DENIS R. HIRSCHFELDT

(Communicated by Carl G. Jockusch, Jr.)

\begin{abstract}
We show that for every computable tree $\mathcal{T}$ with no dead ends and all paths computable, and every $D>_{T} \emptyset$, there is a $D$-computable listing of the isolated paths of $\mathcal{T}$. It follows that for every complete decidable theory $T$ such that all the types of $T$ are computable and every $D>_{T} \emptyset$, there is a $D$-decidable prime model of $T$. This result extends a theorem of Csima and yields a stronger version of the theorem, due independently to Slaman and Wehner, that there is a structure with presentations of every nonzero degree but no computable presentation.
\end{abstract}

\section{INTRODUCTION}

There have been several recent examples of constructions that cannot be performed computably, but can be performed $D$-computably for any noncomputable $D$ (or every noncomputable $D$ in a particular class, such as the $\Delta_{2}^{0}$ sets). A wellknown instance of this phenomenon is due independently to Slaman [8] and Wehner 9. A presentation of a countable structure $\mathcal{M}$ is a structure $\mathcal{A} \cong \mathcal{M}$ with universe $\omega$. The degree of $\mathcal{A}$ is the Turing degree of the atomic diagram of $\mathcal{A}$.

1.1. Theorem (Slaman; Wehner). There is a structure with presentations of every nonzero degree but no computable presentation.

In Corollary 2.5 below, we show that a strong version of this result can be obtained by considering prime models of complete decidable theories all of whose types are computable. Thus this paper fits into an ongoing program of fine analysis of the computable content of Vaughtian model theory (that is, the study of special models such as prime, homogeneous, and saturated models), which includes work by Csima [1] Csima, Hirschfeldt, Knight, and Soare [3] and Csima, Harizanov, Hirschfeldt, and Soare [2. One of the themes of this program is that the modeltheoretic properties of a special model $\mathcal{M}$ can often be used to build a copy $\mathcal{A}$ of $\mathcal{M}$ with some desired computability-theoretic property without explicitly defining an isomorphism between $\mathcal{M}$ and $\mathcal{A}$. This theme is reflected in Theorem 2.1 below and its corollaries.

Received by the editors November 17, 2004.

2000 Mathematics Subject Classification. Primary 03C57, 03D45.

This research was partially supported by NSF Grant DMS-02-00465.

The author thanks Robert Soare for many enlightening discussions related to this paper.

(C)2005 American Mathematical Society 
Another example of a "barely noneffective" construction can be obtained from the following results of Csima [1 and of Goncharov and Nurtazin 4] and independently Millar [7. For a set $D$, a structure $\mathcal{A}$ is $D$-decidable if the elementary diagram of $\mathcal{A}$ is $D$-computable.

1.2. Theorem (Csima). Let $T$ be a complete decidable theory such that all the types of $T$ are computable and let $D$ be such that $\emptyset<_{T} D \leqslant_{T} \emptyset^{\prime}$. Then $T$ has a $D$-decidable prime model.

1.3. Theorem (Goncharov and Nurtazin; Millar). There is a complete decidable theory $T$ such that all the types of $T$ are computable but $T$ has no computable prime model.

1.4. Corollary. There is a complete decidable theory $T$ such that all the types of $T$ are computable and

(1) T has no computable prime model but

(2) $T$ has a D-decidable prime model for every $D$ such that $\emptyset<_{T} D \leqslant_{T} \emptyset^{\prime}$.

It is natural to ask whether Theorem 1.2 can be extended to all noncomputable $D$. We give a positive answer to this question in Corollary 2.3 below.

In [1, Theorem 1.2 was obtained as a corollary to the following omitting types theorem.

1.5. Theorem (Csima). Let $T$ be a complete decidable theory, let $S$ be a uniformly computable set of partial types of $T$, and let $D$ be such that $\emptyset<_{T} D \leqslant_{T} \emptyset^{\prime}$. Then $T$ has a D-decidable model that omits all nonprincipal types in $S$.

The extension of this result to non- $\Delta_{2}^{0}$ degrees is quite different from that of Theorem 1.2. Theorem 1.5 can be extended to all $D$ of hyperimmune degree, but there is a complete decidable theory $T$ and a uniformly computable set $S$ of partial types of $T$ such that any model of $T$ that omits all nonprincipal types in $S$ has hyperimmune degree. These results and their reverse-mathematical consequences will appear in an upcoming paper by Csima, Hirschfeldt, and Shore.

There is a tight connection between complete decidable theories and computable trees with no dead ends. On the one hand, if $T$ is a complete decidable theory, then for each $n$ the tree $S_{n}(T)$ of $n$-types of $T$ is a computable tree with no dead ends, and these trees can be glued together to form a single tree $\mathcal{S}(T)$. On the other hand, any computable tree with no dead ends can be coded into a complete decidable theory. For more details, see Harizanov [5].

The following definition captures those trees that correspond to theories whose types are all computable. (Here $[\mathcal{T}]$ is the set of all paths of the tree $\mathcal{T}$.)

1.6. Definition. A tree $\mathcal{T} \subset 2^{<\omega}$ is a $P A C$ tree if it is a computable tree with no dead ends and every path in $[\mathcal{T}]$ is computable. (PAC stands for paths all computable.)

There is also a tight connection between the effectiveness of prime models of a complete decidable atomic theory $T$ and the effectiveness of listings of the principal types of $T$, and hence with the effectiveness of listings of the isolated paths of $\mathcal{S}(T)$.

1.7. Definition. Let $S \subset 2^{\omega}$ and let $D$ be a set. A $D$-computable listing of $S$ is a uniformly $D$-computable sequence $f_{0}, f_{1}, \ldots \in 2^{\omega}$ such that $S=\left\{f_{n}: n \in \omega\right\}$. (Note that it might be the case that $f_{n}=f_{m}$ for some $n \neq m$.) 
If $S$ is a set of types of some theory $T$, then the concept of a $D$-computable listing of $S$ can be defined analogously.

1.8. Theorem (Goncharov and Nurtazin [4]; Harrington [6]). Let $T$ be a complete decidable theory and let $D$ be a set. Then $T$ has a $D$-decidable prime model if and only if there is a D-computable listing of the principal types of $T$, or equivalently, if and only if there is a D-computable listing of the isolated paths in $[\mathcal{S}(T)]$.

Theorem 1.3 can be restated in terms of PAC trees as follows.

1.9. Theorem (Goncharov and Nurtazin; Millar). There is a PAC tree $\mathcal{T}$ such that there is no computable listing of the isolated paths in $[\mathcal{T}]$.

\section{NEW RESULTS}

The following theorem has a simple proof, but as we will see, it can be combined with some of the results mentioned in the previous section to yield several interesting corollaries, including an extension of Theorem 1.2 and a new, simple proof of a strong form of Theorem 1.1 .

2.1. Theorem. Let $\mathcal{T}$ be a PAC tree and let $D>_{T} \emptyset$. There is a D-computable listing of the isolated paths in $[\mathcal{T}]$.

Proof. Let $\sigma_{0}, \sigma_{1}, \ldots$ be a computable enumeration of the elements of $\mathcal{T}$. We build a $D$-computable listing $f_{0}, f_{1}, \ldots$ of the isolated paths in $[\mathcal{T}]$ so that $\sigma_{n} \subset f_{n}$ for all $n$. Note that if $g$ is an isolated path in $[\mathcal{T}]$, then there is some $n$ such that $g$ is the unique extension of $\sigma_{n}$ in $[\mathcal{T}]$, so if $\sigma_{n} \subset f_{n}$ and $f_{n}$ is a path in $[\mathcal{T}]$, then $f_{n}=g$. So by ensuring that $\sigma_{n} \subset f_{n} \in[\mathcal{T}]$ for all $n$, we ensure that every isolated path in $[\mathcal{T}]$ is on our listing. Thus our only problem is to guarantee that each $f_{n}$ is isolated.

The intuitive idea is to use $D$ to pick the path $f_{n}$. We extend $\sigma_{n}$ until we find a split on $\mathcal{T}$ (if ever). We continue along the right node of this split if $0 \in D$, and along the left node if $0 \notin D$. We then proceed along $\mathcal{T}$ until we find another split (if ever). We continue along the right node of this split if $1 \in D$, and along the left node if $1 \notin D$. We continue defining $f_{n}$ along $\mathcal{T}$ in this manner, using $D$ to choose which direction to take every time we hit a fork in the road. This ensures that $f_{n}$ is $D$-computable (and indeed, that all $f_{n}$ are uniformly $D$-computable). But it also ensures that if we hit infinitely many splits along the way while defining $f_{n}$, then $D$ can be computed from $f_{n}$, which is impossible since $f_{n}$ is in $[\mathcal{T}]$ and hence is computable, and $D$ is not computable. Thus there are only finitely many splits along $f_{n}$, and hence $f_{n}$ is an isolated path.

The formal definition of $f_{n}$ is by recursion. Let $m_{n}^{0}=0$ and $\tau_{n}^{0}=\sigma_{n}$. (The counter $m_{n}^{i}$ keeps track of how many splits have been encountered by step $i$ of the definition.) If $\tau_{n}^{i}$ has only one immediate successor $\mu$ on $\mathcal{T}$, then let $\tau_{n}^{i+1}=\mu$ and $m_{n}^{i+1}=m_{n}^{i}$. Otherwise, let $\tau_{n}^{i+1}=\tau_{n}^{i \bumpeq} D\left(m_{n}^{i}\right)$ and $m_{n}^{i+1}=m_{n}^{i}+1$.

Let $f_{n}=\bigcup_{i} \tau_{n}^{i}$. It is clear that the $f_{n}$ are uniformly $D$-computable, and each $f_{n} \in[\mathcal{T}]$. Also, $\sigma_{n} \subset f_{n}$ for all $n$, and hence, as explained above, every isolated path in $\mathcal{T}$ is $f_{n}$ for some $n$. So all that is left to show is that each $f_{n}$ is isolated.

Suppose that $f_{n}$ is not isolated. Then there are infinitely many $i$ such that $\tau_{n}^{i}$ has two immediate successors. Let $i_{0}<i_{1}<\cdots$ be all such $i$. Note that this list is computable, and $m_{n}^{i_{k}}=k$ for all $k$. Furthermore, for each $k$, the last element of $\tau_{n}^{i_{k}+1}$ is $D\left(m_{n}^{i_{k}}\right)=D(k)$. Thus $D \leqslant_{\mathrm{T}} f_{n}$. But $T$ is a PAC tree, so $f_{n} \leqslant_{\mathrm{T}} \emptyset$, and hence $D \leqslant_{\mathrm{T}} \emptyset$, contradicting the choice of $D$. 
Applying Theorem 1.9, we obtain the following corollary.

2.2. Corollary. There is a PAC tree $\mathcal{T}$ such that there is no computable listing of the isolated paths of $T$, but for every $D>_{T} \emptyset$, there is a D-computable listing of the isolated paths of $T$.

By Theorem 1.8, the following extension of Theorem 1.2 is another consequence of Theorem 2.1

2.3. Corollary. Let $T$ be a complete decidable theory such that all the types of $T$ are computable and let $D>_{T} \emptyset$. Then $T$ has a $D$-decidable prime model.

Combining this result with Theorem [1.3, we obtain the following corollaries, which extend Corollary 1.4 and Theorem [1.1, respectively.

2.4. Corollary. There is a complete decidable theory $T$ such that all the types of $T$ are computable and

(1) T has no computable prime model but

(2) $T$ has a $D$-decidable prime model for every $D>_{T} \emptyset$.

2.5. Corollary. There is a structure $\mathcal{A}$ that has no computable presentation but has a $D$-decidable presentation for every $D>_{T} \emptyset$. Furthermore, $\mathcal{A}$ is the prime model of a complete decidable theory $T$ such that all the types of $T$ are computable.

One way in which the structures built by Slaman and by Wehner in proving Theorem 1.1 differ is that Wehner's structure is elementarily equivalent to a computable structure, while Slaman's is not. The structure in Corollary 2.5] is thus more similar to Wehner's structure than to Slaman's, since it is a model of a complete decidable theory, and hence is elementarily equivalent to a decidable structure.

\section{REFERENCES}

[1] B. F. Csima, Degree spectra of prime models, J. Symbolic Logic 69 (2004) 430-442. MR2058182

[2] B. F. Csima, D. R. Hirschfeldt, V. S. Harizanov, and R. I. Soare, Bounding homogeneous models, to appear.

[3] B. F. Csima, D. R. Hirschfeldt, J. F. Knight, and R. I. Soare, Bounding prime models, J. Symbolic Logic 69 (2004), 1117-1142. MR2135658

[4] S. S. Goncharov and A. T. Nurtazin, Constructive models of complete decidable theories, Algebra and Logic 12 (1973) 67-77. MR0398816 (53:2667)

[5] V. S. Harizanov, Pure computable model theory, in Handbook of Recursive Mathematics (Yu. L. Ershov, S. S. Goncharov, A. Nerode, and J. B. Remmel, eds.), Stud. Logic Found. Math. 138-139 (1998), Elsevier Science, Amsterdam, 3-114. MR.1673621 (2000f:03108)

[6] L. Harrington, Recursively presentable prime models, J. Symbolic Logic 39 (1974) 305-309. MR0351804(50:4292)

[7] T. S. Millar, Foundations of recursive model theory, Ann. Math. Logic 13 (1978) 45-72. MR0482430 (80a:03051)

[8] T. A. Slaman, Relative to any nonrecursive set, Proc. Amer. Math. Soc. 126 (1998) 2117-2122. MR.1443408 (98h:03047)

[9] S. Wehner, Enumerations, countable structures, and Turing degrees, Proc. Amer. Math. Soc. 126 (1998) 2131-2139. MR1443415 (98h:03059)

Department of Mathematics, The University of Chicago, 5734 S. University Ave., Chicago, Illinois 60637

E-mail address: drh@math.uchicago.edu 